\title{
Consequences of electron reflection back upstream in oblique shocks
}

\author{
Paul J. Morris, ${ }^{a, *}$ Artem Bohdan ${ }^{a}$ and Martin Pohl ${ }^{a, b}$ \\ ${ }^{a}$ DESY, 15738 Zeuthen, Germany \\ ${ }^{b}$ Institute of Physics and Astronomy, University of Potsdam, 14476 Potsdam, Germany \\ E-mail: paul.morris@desy.de
}

\begin{abstract}
Astrophysical shocks are believed to efficiently accelerate charged particles, yet electrons need to undergo pre-acceleration to be energetic enough to cross the shock and join the game of acceleration. Understanding the mechanisms responsible for electron pre-acceleration is crucial to solving the shock injection problem. Here, we present PIC simulations of Oblique shocks of varying obliquity angle, using $\theta_{B n}=30^{\circ}, 45^{\circ}, 55^{\circ}, 63^{\circ}$ and $74.3^{\circ}$. Our analyses focus on the reflection of incident electrons back upstream, with these particles capable of generating upstream turbulence and transferring energy away from the shock itself and to the upstream plasma. In this work, we demonstrate that electron reflection initially occurs in the foot region of the shock, with upstream electrons trapped and accelerated by Buneman waves before being reflected. We show that while the proportion of reflected electrons is negligible for $\theta_{B n}=74.3$, but increases to $\sim 5 \%$ for $\theta_{B n}=30$. We show that the most probable energy of reflected electrons is $\propto 1 / \cos \theta_{B n}$, but higher reflection rates at low $\theta_{B n}$ mean in total reflected electrons here carry more kinetic energy back upstream, which produces turbulence. We show that reflected electrons generate electrostatic waves in the upstream region on length scales comparable to Buneman waves, and discuss how these waves interact with upstream electrons, and discuss whether they could compromise the efficiency of electron injection at the shock.
\end{abstract}

$37^{\text {th }}$ International Cosmic Ray Conference (ICRC 2021)

July 12th - 23rd, 2021

Online - Berlin, Germany

\footnotetext{
${ }^{*}$ Presenter
} 


\section{Introduction}

Astrophysical shocks occur ubiquitously in our universe, with detections of non-thermal emission from sources such as supernova remnants implying the presence of accelerated electrons. Diffusive shock acceleration [1] is widely accepted to be the primary acceleration mechanism, yet a pre-requisite for is is that the larmor radius of a particle needs to be comparable to the shock width, which is not the case for electrons at thermal energies. It follows that they therefore require some pre-acceleration, and our physical understanding of it is essential to fully comprehend many astrophysical phenomena.

To understand any electron-scale phenomena responsible for pre-acceleration, we require a method capable of resolving these small kinetic scales. Particle-in-cell (PIC) simulations fulfil this criterion and have previously helped to establish the contribution to electron pre-acceleration in perpendicular shocks from mechanisms such as shock surfing acceleration, magnetic reconnection and stochastic Fermi acceleration [2-6].

An important parameter which heavily influences the behaviour of shock is the obliquity angle, $\theta_{B n}$, which is the angle between the shock normal and the upstream magnetic field [7]. While perpendicular shocks (where $\theta_{B n}=90^{\circ}$ ) typically have well defined shock transitions with a narrow shock foot, in oblique shocks reflected particles can escape upstream along the magnetic field lines, creating an extended region known as the foreshock [8]. Any particles upstream of an oblique shock must encounter the foreshock before reaching the shock, therefore its properties are important as any turbulence in this region has the opportunity to influence upstream plasma before it can encounter and be accelerated at the shock.

In this work, we investigate the impact of the obliquity angle $\theta_{B n}$ on the electron foreshock. We do this by quantifying how many electrons are reflected in each case and discuss how they, and the energy they carry, affects the upstream region.

\section{Simulation setup}

We undertake PIC simulations utilising code developed from TRISTAN [10, 11]. The code is optimised and allows for fully-relativistic $2 \mathrm{D} 3 \mathrm{~V}$ simulations, where tracking two spatial and all three Cartesian components of particle position and velocity, respectively. Such configurations have been shown to reliably reproduce the relevant physics captured in more expensive full 3D simulations $[2,9]$.

Our simulations generate a single shock via reflecting an incoming plasma beam off a conducting wall $[13,14]$, which we define to be at $x=0$. The bulk speed of our incident plasma beam is initially aligned with the negative $x$-axis, with velocity corresponding to $v_{\text {up }}=-0.2 c$ in the simulation frame (giving a shock speed of $v_{\mathrm{sh}}=0.264 \mathrm{c}$ in the upstream frame), and propagates in the $+x$-direction after reflection while the $y$ - and $z$-velocity components remain unaltered. To prevent an initial transient caused by this artificial contact discontinuity, we introduce a drift current to ions close to the reflecting boundary [15], which is removed after reflection has occurred.

In the simulations presented here, inflowing plasma is initialised at all points with an in-plane magnetic field, with $B_{0}=0.451$, with fresh plasma continuously injected from the right boundary of the simulation box. We use an in-plane magnetic field configuration (conventionally $\phi=0$ ), 


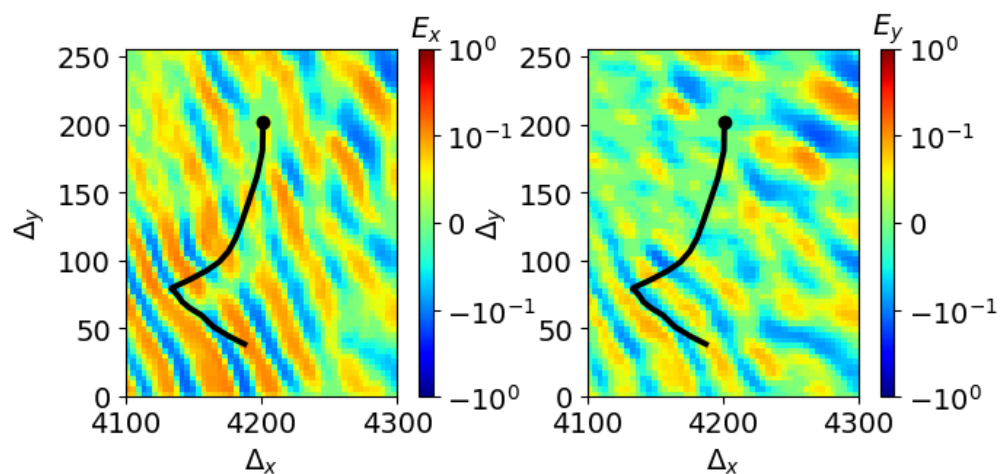

(a)

(b)

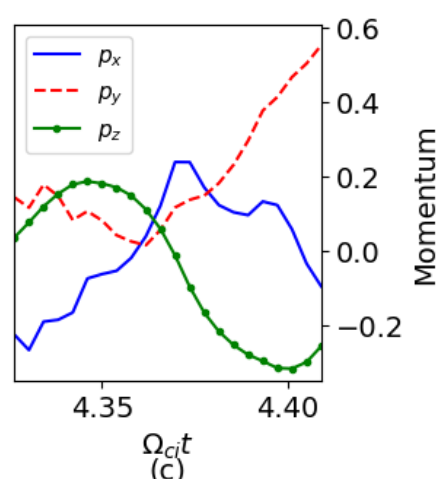

$\Omega_{c i} t$

Figure 1: The trajectory of a reflected electron (black line in panels (a) and (b)) against $E_{x}$ (panel (a)) and $E_{y}$ (panel (b)). Here, an incoming upstream electron becomes trapped in the potential well of a Buneman mode and interacts with it. This causes them to gain momentum in the $x$ - and $y$-directions (panel (c), for the time in which the electron moves along the black line), potentially enabling them to outrun the shock and be reflected. The plot displays quantities in the simulation frame for $\theta_{B n}=55$.

but we change the obliquity angle, using $\theta_{\mathrm{Bn}}=[30,45,55,63,74.3]$ degrees, and compare the properties of the electron foreshocks generated in each case. While still sublimunal, we expect the latter value of $\theta_{B n}=74.3^{\circ}$ to produce few reflected electrons as it is close to the critical angle of $\theta_{\text {crit }}=\tan ^{-1}\left(c / v_{\mathrm{sh}}\right) \sim 75.2^{\circ}$. Its inclusion allow us to directly compare our results with full 3D simulations in the literature, from which we use otherwise identical parameters $\left(M_{A}=21, \beta=1\right.$, $\left.m_{i} / m_{e}=64\right)$ [9].

\section{Results and Discussion}

\subsection{Electron reflection}

Previous work has established the importance of Buneman waves with regards to electron pre-acceleration in high Mach number shocks [5]. They are first excited by an interaction between shock-reflected ions and incoming electrons, and occur at the leading edge of the shock foot, thus can be encountered by upstream electrons before they interact with the shock. They are electrostatic waves which can trap electrons and accelerate them in the associated motional electric field [12]. They occur on spatial scales of,

$$
\lambda_{B}=\frac{2 \pi}{c} \Delta v \lambda_{\mathrm{se}} \sim \frac{2 \pi}{c} v_{\mathrm{sh}} \lambda_{\mathrm{se}}
$$

where $\lambda_{\text {se }}$ is the electron skin length and $\Delta v$ is the relative speed between incoming upstream electrons and reflected ions. For our simulations, $v_{\mathrm{sh}}=0.264 c$, therefore $\lambda_{B} \sim 1.67 \lambda_{\mathrm{se}}$.

Fig. 1 verifies the role of Buneman waves in reflecting electrons at the shock, and shows the case of a typical upstream electron. Here, an upstream electron enters the potential well of an electrostatic Buneman wave, and is carried along with it gaining energy via shock surfing acceleration. To be trapped in the well, the electron initially needs a small momentum across the spatial extent of the well, and it can only escape via Larmor rotation, thus escape becomes more likely at higher energies and Larmor radii larger than the Buneman wave. We typically find that 


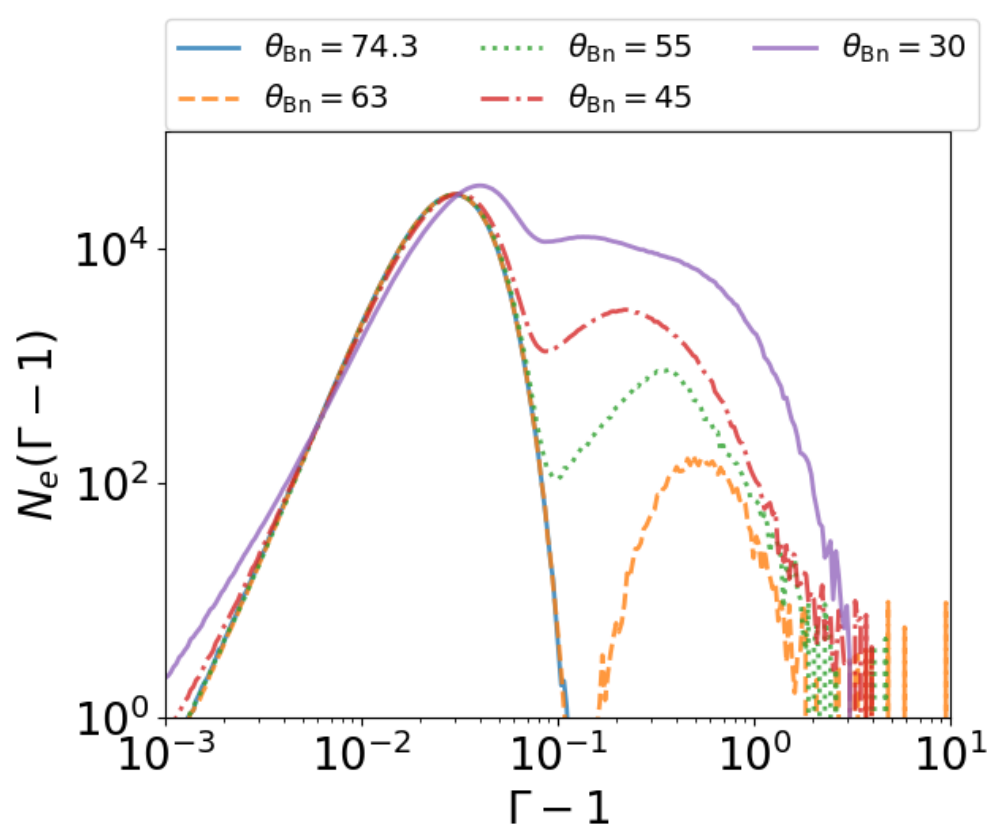

Figure 2: Electron spectra for simulations of varying $\theta_{B n}$ after $\Omega_{c i} t=7.8$. The spectra shown here are measured in the simulation frame. The left hand peak represents the upstream electrons, while electrons at higher values of $\Gamma-1$ have been reflected back upstream. Spectra are computed for a region 15 ion skin lengths $\left(15 \lambda_{s i}\right)$ in the $x$-direction which begins $5 \lambda_{s i}$ upstream from the point where $B / B_{0}=$ 1.02. The full transverse width of the box is used.

more energetic than average electrons are favourably accelerated and reflected by this mechanism, as their larger transverse momenta allows them to more easily enter the potential well of the Buneman mode.

\subsection{Spectra and Reflection Rate}

For each simulation, we compute the electron spectra in the electron foreshock. To ensure consistency between the regions selected for each simulation, we define this region as beginning (in simulation box coordinates) at $x=x\left(B / B_{0}=1.02\right)+5 \lambda_{\mathrm{si}}$ and ending at $x=x\left(B / B_{0}=1.02\right)+20 \lambda_{\mathrm{si}}$ $\left(\lambda_{\mathrm{si}}=200 \Delta\right.$ and is the ion skin length) where $x\left(B / B_{0}=1.02\right)$ is the closest point to the rightmost boundary of the simulation box (in the upstream) for which $B / B_{0} \geq 1.02$. Whilst this choice appears arbitrary, it is necessary to ensure we only consider upstream and reflected electrons, as if we are too close to the shock other energetic electrons which would eventually pass downstream are included. Similarly, if this region is too large, we risk including regions of the simulation box which contain no reflected electrons because they have not yet had time to travel this far.

Fig. 2 shows the electron spectra for this region after the simulation has reached $\Omega_{\mathrm{ci}} t=7.8$. Two features immediately stand out. Firstly, more electrons are reflected for smaller values of $\theta_{B n}$, and secondly, the peak value of $\Gamma-1$ shifts to higher values, corresponding to higher electron energies, for larger $\theta_{B n}$. The physical interpretation of these features are interconnected. Previous work has demonstrated that electrons arriving at the shock can be reflected back upstream if their velocity satisfies $v_{e} \cos \theta_{B n} \geq v_{\mathrm{sh}}[9,17]$. This result is intuitive when one considers that reflected electrons travel in the direction of the upstream magnetic field lines, and can only be reflected if they can outrun the shock. In the simulations presented here, we fix $v_{\text {sh }} \approx 0.264 c$, thus on average, to be reflected, electrons require larger $v_{e}$ for larger values of $\theta_{B n}$. Accordingly, the relative abundance of lower energy electrons means more of them fufill this criteria for lower values of $\theta_{B n}$, hence a greater proportion are reflected. Similarly, as for larger values of $\theta_{B n}$ only electrons with a larger 


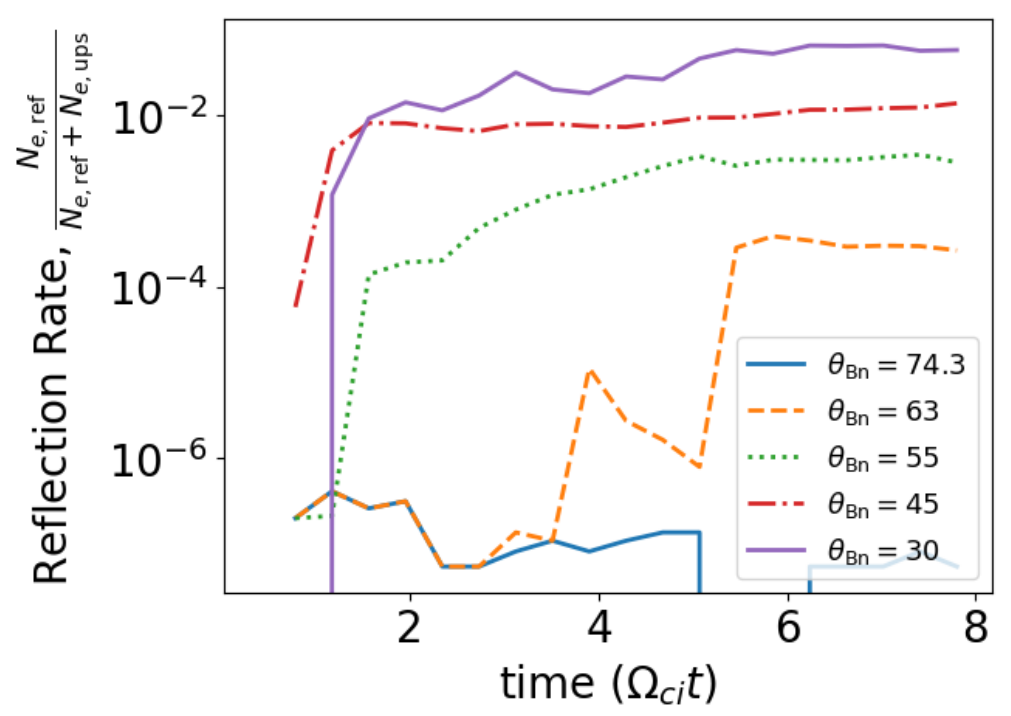

Figure 3: Electron reflection rates for the different simulations as function of time in units of ion gyroperiods. When $\theta_{B n}$ is smaller, more electrons are reflected and the foreshock region is more extended.

$v_{e}$ can be reflected, the peak value of $\Gamma-1$ increases as is shown in Fig. 2. We also find that faster electrons are located at the front of the foreshock because they can more easily outrun the shock.

We can estimate the reflection rate in the regions over which our spectra were calculated by making a cut in $\Gamma-1$, and considering electrons with values above this threshold to be reflected, while those below are moving with the upstream bulk flow. For this, we use $(\Gamma-1)_{\text {cut }}=3 \times 10^{-2}$. The reflection rate is then given by the ratio of reflected to total (reflected + upstream) electrons, $R=N_{e \text {,ref }} /\left(N_{e, \text { ref }}+N_{e \text {,ups }}\right)$ The reflection rate as a function of time is shown in Fig. 3. As expected from the spectra above, simulations with lower $\theta_{B n}$ have larger reflection rates, with the highest rates being a few percent for $\theta_{B n}=30^{\circ}$. Additionally, the onset of reflection begins at later time for larger $\theta_{B n}$, with this effect particularly noticeable for $\theta_{B n}=63$. This is because for larger $\theta_{B n}$ more time is required to accelerate electrons up to sufficient energies such that they can outrun the shock and be reflected upstream.

\subsection{Effect on the Upstream Plasma}

Of particular relevance in the context of astrophysical shocks is how much kinetic energy these reflected electrons can carry back upstream and whether it can drive any turbulence that affects the upstream plasma. Reflected electrons, of Lorentz factor $\Gamma$, have a kinetic energy given by $E_{K}=(\Gamma-1) m_{e} c^{2}$. Previously, we established that shocks with smaller $\theta_{B n}$ reflect a greater number of electrons, but with lower average energy. Here, we estimate the total energy content carried in the reflected component relative to the upstream medium. One can estimate the total fraction of reflected energy by calculating,

$$
\frac{E_{\mathrm{ref}}}{E_{0}}=R_{\max }\left[\frac{\overline{(\Gamma-1)_{\text {ref }}}}{\overline{(\Gamma-1)_{\text {ups }}}}\right],
$$

where $R_{\max }$ is taken as the maximum reflection rate from Fig. 3, and the term in the square brackets is the ratio between the mean values of $(\Gamma-1)$ for our reflected and upstream electrons defined 


\begin{tabular}{c|c|c|c|c}
$\theta_{B n}$ & 30 & 45 & 55 & 63 \\
\hline$E_{\text {ref }} / E_{0}$ & $5.38 \times 10^{-2}$ & $1.1 \times 10^{-2}$ & $3.79 \times 10^{-3}$ & $6.87 \times 10^{-4}$
\end{tabular}

Table 1: The fraction of energy carried back upstream by reflected electrons relative to those propagating upstream towards the shock as a function of $\theta_{B n}$. More energy in total is carried back upstream by the larger number of reflected lower energy electrons in simulations with smaller $\theta_{B n}$.

previously. The results are shown in Table 1 , from which it is clear that a smaller $\theta_{B n}$ results in significantly more energy carried back upstream by these reflected electrons.

We now turn our attention to understanding what happens to this reflected energy content, and what turbulence it can drive in the upstream region. This is particularly important, as any impact on the incoming upstream electrons could potentially compromise their injection into the shock. From our simulations, we note the presence of electrostatic waves in the $x y$ plane of our simulation box over the same spatial extent where reflected electrons are present. To demonstrate that these waves are driven by reflected electrons, and to establish their energy content, we perform a 2-dimensional discrete Fourier transform (DFT) over the simulation box where they are present. Following [16], the 2D dimensionless Fourier amplitudes are computed according to,

$$
\widetilde{E}\left(k_{m, n}\right)=\frac{e}{m_{e} c \omega_{p}} \frac{1}{N_{x} N_{y}} \sum_{i=0}^{N_{x}-1} \sum_{j=0}^{N_{y}-1} \boldsymbol{e}_{k} \cdot \boldsymbol{E}\left(x_{i}, y_{j}\right) \exp \left(2 \pi i\left[\frac{m i}{N_{x}}+\frac{n j}{N_{y}}\right]\right),
$$

where $m$ and $n$ denote the respective indices of the $x$ and $y$ wave-vector components, and $\boldsymbol{e}_{\boldsymbol{k}}$ is in the direction of the unit wave vector. From this, using Parseval's theorem, the energy density of the electrostatic field can be calculated by integrating over $\left|\widetilde{E}\left(k_{m, n}\right)\right|^{2}$.

Fig. 4 shows the DFT over a region containing electrostatic waves. The peak power corresponds to a spatial scale of $\lambda_{\mathrm{ES}} \sim 3 \lambda_{\mathrm{se}}$, which further supports that these waves are driven by electrons as they occur on electron length scales comparable to our estimated wavelength of Buneman waves of $\lambda_{B} \sim 1.7 \lambda_{\mathrm{se}}$.

Finally, using Parseval's theorem, we compute the total energy in reflected electrons in a DFT window which we slide across the upstream region in our simulation box. This window is square in shape of size $40 \lambda_{\mathrm{se}} \times 40 \lambda_{\mathrm{se}}$ to ensure appropriate sampling of the electrostatic mode. We compute this only for the upstream region (i.e. $\left.x>x\left(B / B_{0}=1.02\right)+5 \lambda_{\mathrm{si}}\right)$ and compute the percentage of reflected electrons within the same window using the same value of $(\Gamma-1)_{\text {cut }}$ as before.

Fig. 5 plots the total energy density contained in these electrostatic waves as a function of the electron reflection rate. It is clear that when an extended foreshock is present, a strong correlation exists between the two, indicating that these waves are indeed driven by reflected electrons. Furthermore, our preliminary analyses indicate that, as with Buneman waves, these electrostatic waves can accelerate upstream electrons and even reflect them back upstream. When the foreshock is extended, this process can occur at distances far from the shock, potentially reducing the number of electrons available for diffusive shock acceleration. The reflection of electrons in this manner will again help to drive further upstream turbulence, and contribute to further electron reflection. 


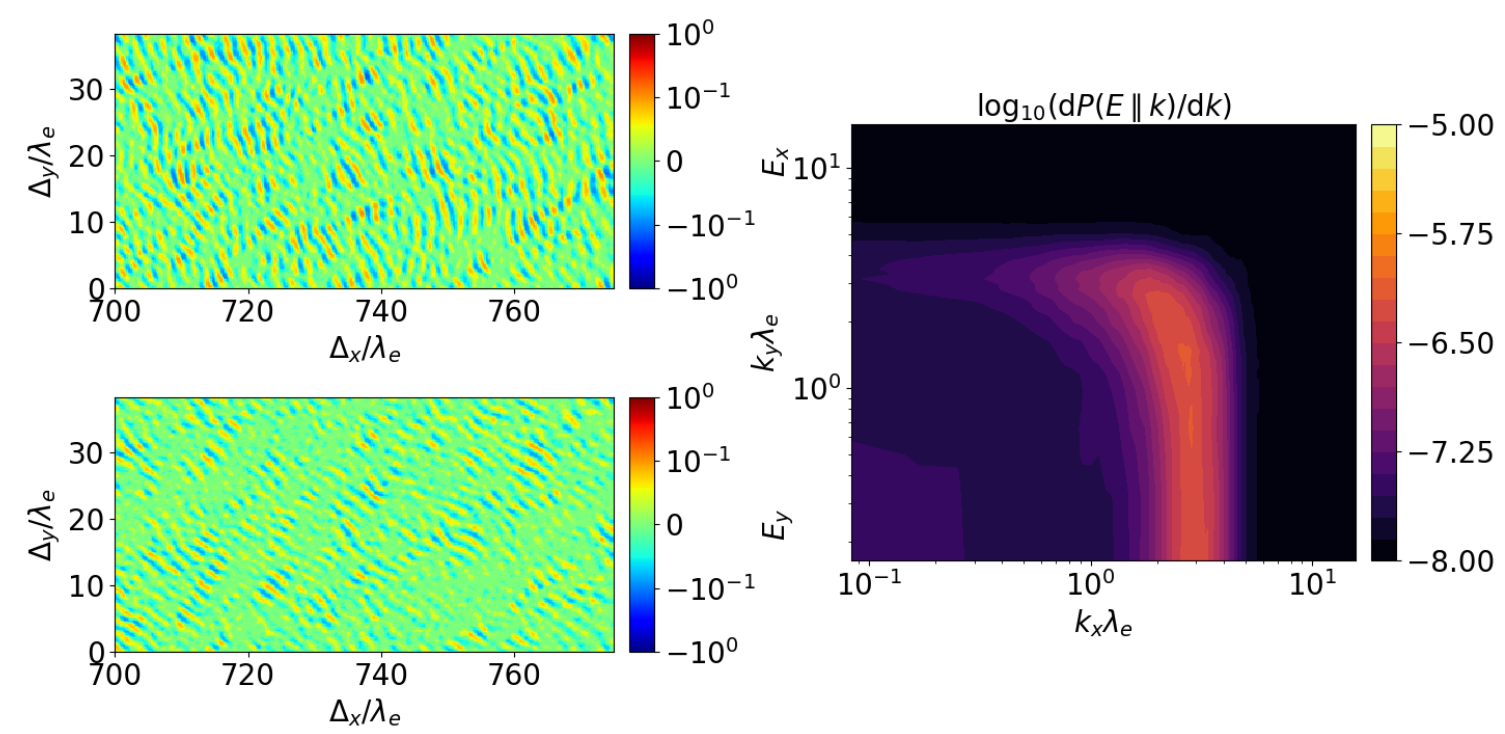

Figure 4: Colour maps in $E_{x}$ (top left) and $E_{y}$ (lower left) show the electrostatic waves generated by reflected electrons in the electron foreshock for the simulation with $\theta_{B n}=30^{\circ}$. The right panel displays the Fourier amplitudes (right) for this region in units of $\left[c /\left(m_{e} c \omega_{p, e}\right)\right]^{2}$, indicating a peak at $k \lambda_{S E} \sim 2$.

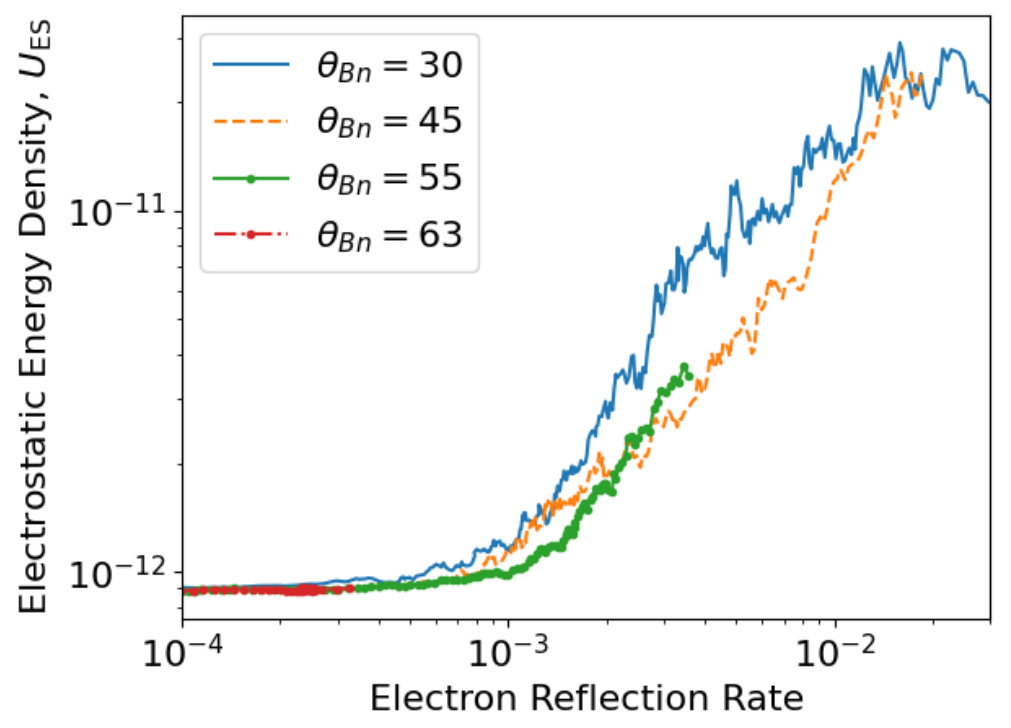

Figure 5: Plot of the energy density of the electrostatic waves generated in the electron foreshock as a function of the electron reflection rate in that region. The strong correlation present in all simulations here supports the view that reflected electrons drive these waves.

\section{Conclusions}

- In-plane PIC simulations were completed for the range $\theta_{B n}=[30,45,55,63,74.3]$ degrees to quantify the rates of incident electrons that are reflected back upstream and assess their effect on the upstream plasma.

- A higher percentage of incoming electrons are reflected back upstream for smaller $\theta_{B n}$. About $5 \%$ of electrons were reflected for $\theta_{B n}=30^{\circ}$, but only $\sim 0.03 \%$ for $\theta_{B n}=63^{\circ}$.

- Although fewer in number, the peak energy of reflected electrons increases with increasing 
$\theta_{B n}$. This is because they require velocities $v_{e} \cos \theta_{B n}>v_{\mathrm{sh}}$. Despite this, the greater number of electrons reflected in shocks with smaller $\theta_{B n}$ means overall more energy is carried back upstream than for larger $\theta_{B n}$.

- These reflected electrons drive electrostatic waves on scales comparable to Buneman waves in the electron foreshock upstream.

- Preliminary analysis indicates that these waves are themselves capable of accelerating and reflecting upstream electrons further away from the shock, preventing them from reaching it and compromising injection.

\section{Acknowledgments}

M.P. acknowledges support by DFG through grant PO 1508/10-1. The numerical experiments were done with HLRN supercomputer at North-German Supercomputing Alliance under the project bbp00033.

\section{References}

[1] Bell, A. R., 1978, MNRAS, 182, 147-156

[2] Bohdan, A., Niemiec, J., Kobzar, O. and Pohl, M., 2017, ApJ, 847, 71

[3] Bohdan, A., et al., 2019, ApJ, 878, 5

[4] Bohdan, A., et al., 2019, ApJ, 885, 10

[5] Bohdan, A., et al., 2020, ApJ, 893, 6

[6] Bohdan, A., et al., 2020, ApJ, 904, 12

[7] Sironi, L., and Spitkovsky, A., 2009, ApJ, 698:2, 1523

[8] Treumann, R. A., 2009, Astron. Astrophys. Rev., 17, 409-535

[9] Matsumoto, Y., Amano, T., Kato, T. N., and Hoshino, M., 2017, PRL, 119, 105101

[10] Buneman, O., Computer Space Plasma Physics: Simulation Techniques and Software Eds.: H. Matsumoto \& Y. Omura, Tokyo: Terra Scientific, 1993

[11] Niemiec, J., Pohl, M., Stroman, T., et al. 2008, ApJ, 684, 1174.

[12] Shimada, N. and Hoshino, M., 2000, ApJL, 543, L67-L71

[13] Quest, K. B., 1985, Phys. Rev. Lett., 54, 1872

[14] Burgess, D., Wilkinson, W. P., Schwartz, S. J., 1989, JGR, 94, 8783

[15] Wieland, V., Pohl, M., Niemiec, J., Rafighi, I. and Nishikawa, K., 1989, ApJ, 62, 820

[16] Vafin, S., Rafighi, I., Pohl, M. and Niemiec, J., 2018, ApJ, 43, 857

[17] Amano, T., and Hoshino, M., 2007, ApJ, 190, 661 